\title{
Perception of the major/minor distinction: III. Hedonic, musical, and affective discriminations
}

\author{
ROBERT G. CROWDER \\ Yale University, New Haven, Connecticut
}

\begin{abstract}
Long sequences of synthetic, sine-wave triads were presented for identification according to three criteria: major/minor, happy/sad, or pleasant/unpleasant. The triads ranged in small steps from the equal-tempered minor to the major mode, and the main performance measure was how identification judgments were affected by the triads' positions along this continuum. The major/minor and happy/sad criteria produced nearly indistinguishable, increasing psychophysical functions; however, judgments of pleasantness followed a different pattern, reaching a minimum halfway between major and minor tunings, perhaps because, at this point, the triads were badly out of tune.
\end{abstract}

In a previous article (Crowder, in press b), I described several experiments on the discrimination and identification of musical triads varying on the major/minor dimension. These triads, prepared from sine waves, formed nine-step continua ranging from a prototype minor chord (for example, E, G, and B) to a prototype major chord (E, G-sharp, and B) in logarithmically equal steps. In the identification experiments, subjects of heterogeneous musical backgrounds indicated whether such items sounded " "major" or "minor." The test triads were presented in random order from three tonalities (E, F, and F-sharp) and sampled in haphazard order the different locations on the nine-step "'majorness"' continua. Examples of the labeled endpoints and of the ordered continua were provided during the instruction period before the formal data collection. The identification results showed a regular, increasing, psychophysical function relating presented and perceived "majorness." In one study (Crowder, in press $b$, Experiment 2), the mean majorness ratingsout of a possible 18-for the 9 steps were 4.50, 4.83, 5.67, $5.72,7.50,10.00,12.17,12.50$, and 13.44. A detailed examination of individual differences showed that some individuals had much steeper psychophysical functions than this, while others had essentially flat ones.

In another article (Crowder, in press a), I explained that the major/minor distinction is more than just one contrast among many that could be selected for psychophysical analysis. The association of terms like "happy" and "sad" with the major and minor modes is possibly the most conventional link of emotional mood with objective musical events that we have in traditional Western music. The two extreme points of view as to the source of this connotation are (1) that it is a product of cultural indoctrination, and (2) that it is inherent in the acoustics of musical sounds (see Crowder, in press a, for a full discussion

This research was supported by NSF Grant BNS 82-19661. I appreciate the assistance of Herta Flor in collecting the data for Experiment 1. of these and other proposed explanations). The present paper does not settle this issue, but it does add pertinent information on how different judgment criteria for these sine-wave triads produce different results.

\section{EXPERIMENT 1}

Separate groups of unselected subjects in this experiment judged the stimuli described above on either a major/ minor (musical) or a happy/sad (affective) criterion. If judgments of major/minor had been mediated by an affective reaction in the previous experiments, then the happy/sad judgment, being more direct, ought to yield better performance (steeper psychophysical functions) than judgments of major/minor. Perhaps people who had difficulty in the earlier identification experiments, people who showed a flat psychophysical function across the nine degrees of majorness, could readily perceive the different affective tones in the two modes but could not make the major/minor distinction.

\footnotetext{
Method

Twenty-four young adults from the Yale summer community served as subjects in return for pay. They were of heterogeneous musical experience, and although musical background was assessed in a formal interview before the experimental session, no interesting relations between musical background and performance were uncovered (beyond those identified in Crowder, in press b). The stimuli were the same 162 sine-wave triads used in one of the earlier identification studies (see Crowder, in press b, Experiment 1, for details of their synthesis.) There were three tonalities (E, F, and F-sharp), two inversions for each chord (root position and first inversion), and nine steps between minor and major for each tonality-inversion combination, resulting in a total of $3 \times 2$ $\times 9=54$ items, each of which was presented three times in completely random order. The chords were $300 \mathrm{msec}$ long and the interstimulus interval was $3 \mathrm{sec}$. Participants were given a numbered answer sheet with columns headed by the words "major" and "minor" (or "happy" and "sad") and spaces for checks in both columns for each of the 162 trials. The critical experimental variable was the instruction for the judgment criterion that should be applied to each triad. The two options are given below. The subject read one of the two sections in brackets, depending on whether the subject was among the 12 assigned to the major/minor condition or the 12 assigned to the happy/sad condition:
} 
This experiment is about your ability to hear very subtle differences among musical chords. Please listen while I play some examples of what these chords sound like. (Experimenter plays 20 randomly selected chords.)

[These chords have been chosen to include a range of different emotional connotations, from "sad" to "happy," with some neutral ones in between. In music theory, these sad and happy chords are referred to as minor and major, respectively, but I want you to focus on their emotional tone. Now listen twice to a series of nine chords that ranges from "pure sad" to "pure happy" in small steps.]

[These chords have been chosen to include a range of harmonic values, from "minor" to "major" with many neutral ones in between. You may not be familiar with the terms minor and major but the distinction is not too difficult. Now listen twice ....]

The remaining instructions were identical for the two groups and pertained to mechanics of the test session.

\section{Results}

The results of Experiment 1 are given in Figure 1. People working on the basis of the affective criterion produced nearly identical results to those working on the basis of the major/minor criterion. An analysis of variance, with type of distinction and degree of majorness as the factors, confirmed this assessment of the data, giving a reliable main effect of majorness $[F(8,176)=13.24$, $p<.01$ ], but no effect of judgmental distinction and no interaction (Fs < 1.0). These results simply indicate that, for whatever reason, the happy/sad and major/minor instructions were equivalent when these procedures were followed. The poor performance of participants in earlier experiments, using the major/minor terminology, apparently cannot be blamed on the unfamiliarity of that terminology compared with the "natural" affective language.

\section{EXPERIMENT 2}

Helmholtz (1885/1954, pp. 214-217) thought the traditional negative connotation of the minor triad could be

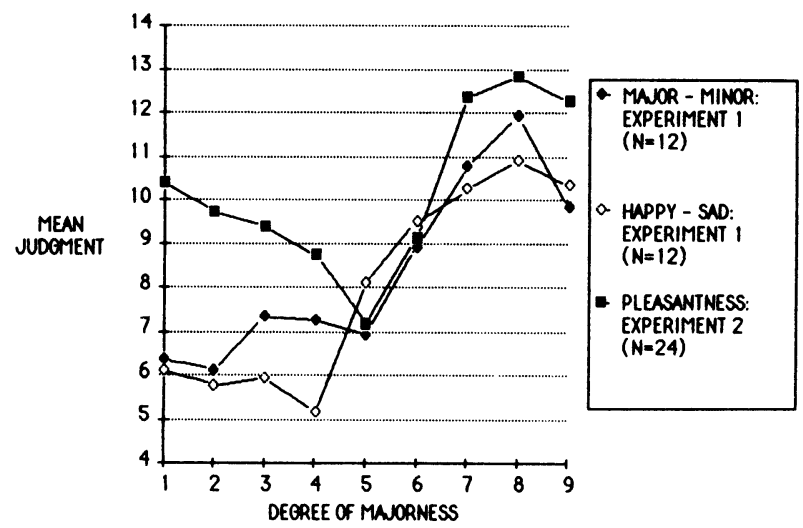

Figure 1. Mean judgments of triads as a function of degree of majorness. Number 1 is a conventional (equal-tempered) minor triad, and number 9 is a major triad. In Experiment 1, people made binary judgments on a major/minor or a happy/sad criterion. In Experiment 2 , the criterion was pleasant/unpleasant. understood in terms of dissonance. The basis for his derivations was the "just" rather than "equal" tuning system; however, we may disregard this detail for purposes of the present experiment, partly because sine-wave stimuli were used here. For real musical tones, Helmholtz argued as follows: Relative to the major triad, the minor triad generates "near-misses" among the harmonics and combination tones, leading to greater beats, roughness, and a generally more dissonant impression. Dissonance, in turn, was plainly a hedonic dimension for Helmholtz: "The nerves of hearing feel these rapid beats as rough and unpleasant ...' (Helmholtz, 1884/1954, p. 226). In support of Helmholtz's position, Roberts (1983) found that both musically sophisticated and musically unsophisticated subjects rated minor chords as more dissonant than major chords. Without presuming to submit Helmholtz's entire reasoning to a critical test, we may ask whether both the harmonic judgments and the affective judgments measured in Experiment 1 actually collapse onto a single hedonic dimension of pleasantness. If so, then perhaps any criterion with an evaluative polarity would yield the same sort of function. This possibility was explored in Experiment 2.

\section{Method}

Twenty-four Yale undergraduates from a course in music psychology were tested in a group. The method was the same as in Experiment 1, with the exceptions of group testing and the following: First, the stimulus tape used three tonalities, as before, but there were three versions of each chord-one in root position, one in the first inversion, and another in root position an octave higher than the first. This variable was included for reasons that are not germane to this report. Second, the subjects were told to make ratings on an answer sheet with columns headed "pleasant" and "unpleasant," rather than "major" and "minor" or "happy" and "sad." No suggestion was given as to what prototype chords would sound like for the pleasant and unpleasant cases; however, examples of the stimuli were played before the beginning of the main session.

\section{Results}

The results of Experiment 2 are shown, along with those of Experiment 1, in Figure 1. The hedonic criterion used as the basis for judgment in this experiment led subjects to produce a V-shaped function with a minimum at the middle position of the nine-step continuum, in contrast to the increasing psychophysical functions that resulted from the two instructions of Experiment 1. The overall main effect of majorness was highly reliable in the present data set, however $[F(8,184), p<.01]$. A spot check confirmed the statistical reliability of the form of the function: A simple sign test was performed comparing positions 1 and 5 on the majorness dimension. With one tie, 19 of the remaining 23 subjects rated the latter position on the dimension as less pleasant than the former $(\mathrm{p}=.001)$.

\section{GENERAL DISCUSSION}

People can make judgments of affective tone or of harmonic mode with approximately equal facility when they are given a majorness continuum and examples of the two endpoints. Comparable subjects with comparable stimuli behave very differently when asked to make hedonic 
(pleasantness) judgments without suggestions for endpoint labels. People making hedonic responses show a strong dislike of the triads that are the farthest from regular, equal-tempered chromatic pitches. Perhaps this "mistuned" quality dominated the judgments of subjects in Experiment 2 . At the least, however, the two experiments show that the majorness and affective judgments must be reflecting more than a single evaluative dimension with positive at one end and negative at the other. To this limited extent, the results comment on Helmholtz's idea that minor triads sound dissonant and therefore produce reactions of distress. This could be the mechanism by which the affective connotations of major and minor first evolved (before the days of equal temperament). However, modern listeners can easily keep straight the triads they know to label as happy and sad, or to label as major and minor, from those triads with sounds they like and dislike. Nevertheless, a full and proper evaluation of Helmholtz's theory will require using triads made from tones with harmonics, rather than sine waves, and possibly tuned to the just temperament.

\section{REFERENCES}

Crowder, R. G. (in press a). Perception of the major/minor distinction: I. Historical and theoretical foundations. Psychomusicology. Crowder, R. G. (in press b). Perception of the major/minor distinction: II. Experimental Investigations. Psychomusicology.

Helmholtz, H. L. F. (1954). On the sensations of tone. New York: Dover. (Original work published 1885.)

RoBerts, L. A. (1983). Consonance and dissonance judgments of musical chords. Paper presented at the 105th meeting of the Acoustical Society of America, Cincinnati, $\mathrm{OH}$.

(Manuscript received for publication March 22, 1985.) 